\title{
Clinicopathological features and endoscopic characteristics of inverted sessile serrated adenomas/polyps
}

\section{다)(1) $\odot$}

Authors

Kenji Takashima ${ }^{*}, 1,2$, Yasuhiro Oono ${ }^{*}, 1$, Motohiro Kojima ${ }^{*}{ }^{3}$, Hiroaki Ikematsu' ${ }^{1}$, Tetsuo Akimoto ${ }^{2,4}$, Tomonori Yano ${ }^{1}$

Institutions

1 Department of Gastroenterology and Endoscopy, National Cancer Center Hospital East, Kashiwa, Chiba, Japan

2 Course of Advanced Clinical Research of Cancer, Juntendo University Graduate School of Medicine, Kashiwa. Chiba, Japan

3 Department of Pathology Division and Research Center for Innovative Oncology, National Cancer Center Hospital East, Kashiwa, Chiba, Japan

4 Department of Radiation Oncology, National Cancer Center Hospital East, Kashiwa, Chiba 277-8577, Japan

submitted 24.5.2018

accepted after revision 22.10.2018

\author{
Bibliography \\ DOI https://doi.org/10.1055/a-0820-2179 | \\ Endoscopy International Open 2019; 07: E330-E336 \\ (c) Georg Thieme Verlag KG Stuttgart · New York \\ ISSN 2364-3722
}

Corresponding author

Kenji Takashima, Endoscopy Division, National Cancer

Center Hospital East, 6-5-1 Kashiwanoha, Kashiwashi, Chiba

277-8577, Japan

Fax: +81-47-134-6928

ktakashi@east.ncc.go.jp

\section{ABSTRACT}

Background and study aims This study was designed to clarify clinicopathological, endoscopic, and genetic characteristics of inverted sessile serrated adenomas/polyps.
Patients and methods In this retrospective study, we reviewed the pathology reports of patients treated in our hospital between January 2010 and June 2015 and identified sessile serrated adenomas/polyps that were diagnosed with endoscopic resected specimens. Clinicopathological, endoscopic, and genetic features were compared between the inverted and ordinary types.

Results Among the 104 sessile serrated adenomas/polyps, 37 lesions were inverted (35.6\%). The inverted types had two patterns of invasion: expansive and infiltrating growth. Expansive growth was observed in 15 cases $(40.5 \%)$ and infiltrating in 22 (59.5\%). Regarding the clinicopathological findings and endoscopic characteristics of the inverted types, presence of adherent mucous, location in the rightside colon, an expanded type-II pit pattern, irregularly dilated vessels, and a depression were demonstrated in $89 \%$ (33/37), 73\% (27/37), 54\% (20/37), 75\% (28/37), and 35\% (13/37), respectively. Contrastingly, these were demonstrated in $86 \%$ (58/67), 84\% (56/67), $55 \%$ (37/67), 58\% $(39 / 67)$, and $0 \%(0 / 67)$, respectively, in the ordinary type. In the univariate analysis, male sex and a depression in the adenoma/polyp were significantly associated with inverted types $(P<0.001)$. BRAF mutation at codon 600 was found in six of seven ordinary types and in 11 of 11 of the inverted types. KRAS, NRAS, and PIK3CA mutations were not found in the ordinary and inverted types.

Conclusion More than $30 \%$ of sessile serrated adenomas/ polyps were pathologically diagnosed as inverted, and approximately $40 \%$ of them were of the expansive growth type and easily diagnosed with endoscopy.

\section{Introduction}

Most colorectal carcinomas (CRCs) originate from adenomas [1]. However, a serrated neoplastic pathway has been recently accepted, in which CRCs develop from serrated lesions of the

\footnotetext{
* These authors contributed equally.
}

colon and rectum. Serrated lesions were initially described by Longacre, and this pathway accounts for as many as $30 \%$ to $35 \%$ of all sporadic CRCs [2,3]. Serrated lesions have been classified into three groups: hyperplastic polyps (HPs), sessile serrated adenomas/polyps (SSA/Ps), and traditional serrated adenomas $[1,4]$. Among these, SSA/Ps constituted $15 \%$ to $25 \%$ of the serrated lesions and have been reported as precursors of sporadic CRCs with microsatellite instability $[5,6]$, BRAF mutation 


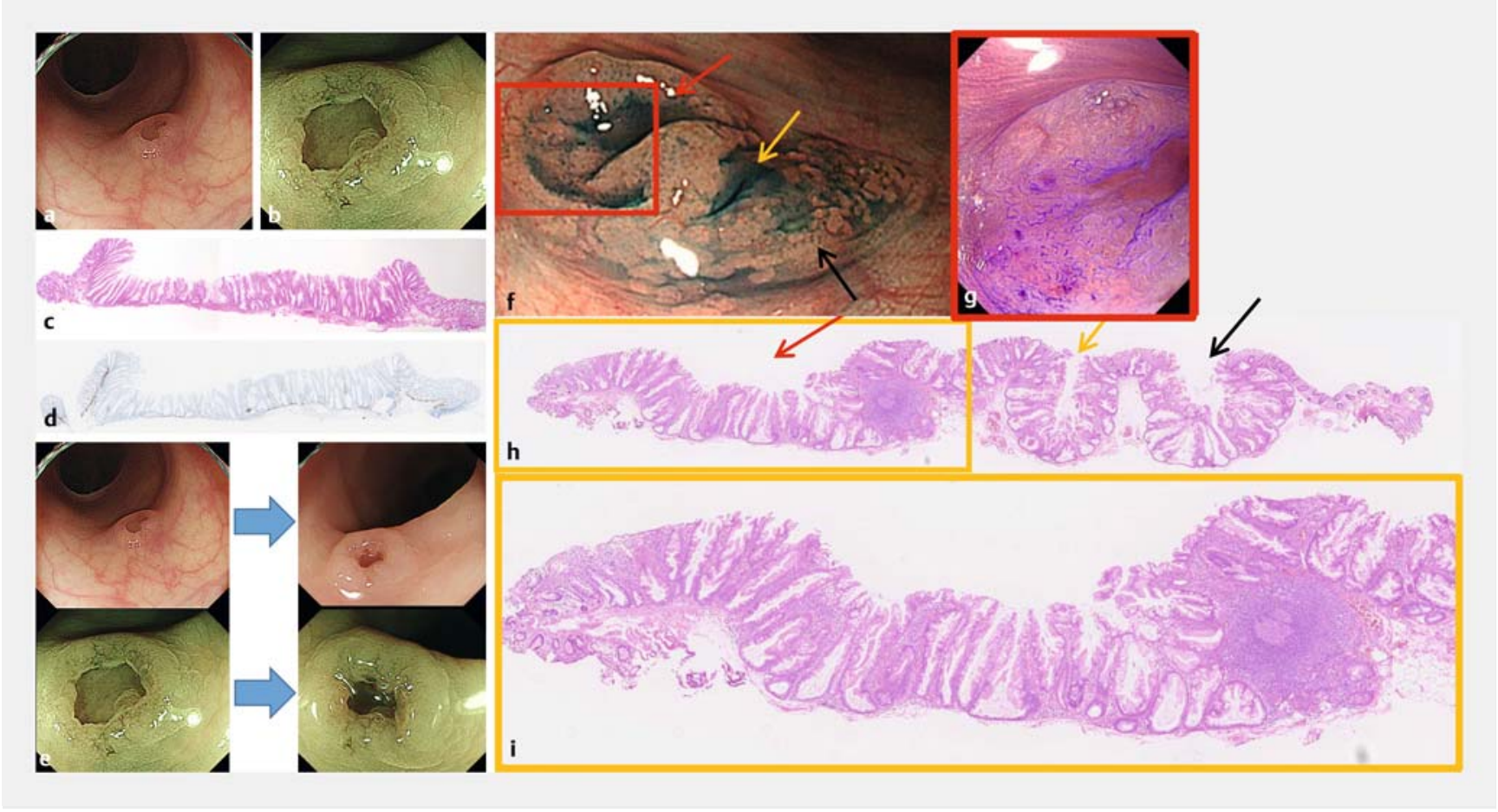

- Fig. 1 Endoscopic and histological features of EGT. a, b Colonoscopy displayed a 6-mm flat-elevated lesion with a central depression in the descending colon. The mucosal central depression was a deep lesion with mucin hypersecretion. NBI with magnification shows VMV. e The morphology of the lesion changed over time. $\mathbf{f}, \mathbf{g}$ Colonoscopy displayed a 10-mm flat-elevated lesion with three clearly demarcated and deep lesions (red and yellow arrows) in the transverse colon. $\mathbf{c}, \mathbf{d}, \mathbf{h}, \mathbf{i}$ EGT exhibits endophytic growth of crypt dilation, irregularly branching crypts, and horizontally arranged basal area crypts with a clear delimited border.

[7], and a CpG island methylator phenotype [8-10]. Therefore, early detection of SSA/Ps is important for prevention of CRCs. We previously published a report of the first identified depressed type of SSA/P, designated as an inverted SSA/P (InSSA/P) [11].

Inverted HPs (InHPs) have been reported to have endophytic growth of the hyperplastic gland $[12,13]$. InSSA/Ps also exhibit the same phenomenon and show a form that is unusual for an SSA/P. However, there have been few reports of their clinicopathological characteristics, endoscopic features, and genetic characteristics. Thus, the aim of this retrospective study was to clarify the clinicopathological characteristics, endoscopic features, and genetic characteristics of InSSA/Ps.

\section{Patients and methods}

The study involved retrospective data collection at an endoscopy unit in the National Cancer Center Hospital East, Kashiwa, Japan. We reviewed pathology reports from our hospital for January 2010 to June 2015 and identified SSA/Ps that were diagnosed with endoscopic resected specimens. Histology reports of these cases were reviewed and clinicopathological characteristics, endoscopic features, and genetic characteristics of InSSA/Ps were compared with those of non-inverted SSA/Ps.

\section{Histopathology}

Histopathological diagnosis of SSA/P was confirmed according to the World Health Organization's 2010 classification [10] and was evaluated by gastrointestinal pathologists. The criteria comprise abnormal architecture of the crypt bases: a so-called abnormal proliferation (such as crypt dilation), irregularly branching crypts, and horizontally arranged basal area crypts (inverted T- and/or -shaped crypts) [10]. InSSA/Ps have forms similar to those of InHPs and exhibit endophytic growth of the SSA/P's gland [11]. SSA/Ps that did not have these characteristics were defined as ordinary-SSA/Ps (O-SSA/Ps) in this study. Selected InSSA/Ps were further divided into two pathological patterns: expansive growth type (EGT) and infiltrating growth type (IGT) [12]. The pathological difference between these two types is as follows. EGT was endophytic growth of crypt dilation, irregularly branching crypts, and horizontally arranged basal area crypts with clear, delimited borders ( $\bullet$ Fig. 1). IGT was an endophytic growth of scattered crypt dilation, irregularly branching crypts, and horizontally arranged basal area crypts with invagination into the submucosal layer or submucosal lymphoid tissue; invagination into the submucosa was always found ( $\bullet$ Fig. 2). 


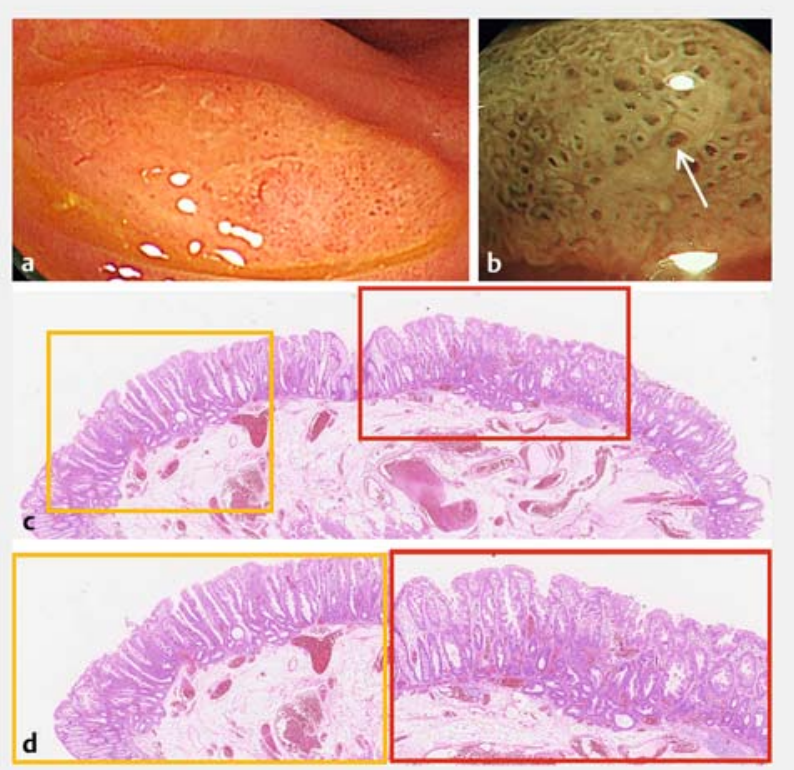

- Fig. 2 Endoscopic and histological features of IGT. a, b Colonoscopy displayed an 8-mm flat-elevated lesion in the ascending colon. It exhibited mucin hypersecretion and NBI with magnification showed dilated type II pit pattern (whites arrows). c, d IGT exhibits endophytic growth of scattered crypt dilation, irregularly branching crypts, and horizontally arranged basal area crypts with the invagination into the submucosal layer.

\section{Colonoscopy}

Endoscopists retrospectively reviewed the colonoscopy images from SSA/Ps diagnosed pathologically between January 2010 and June 2015, and characteristic colonoscopic findings of the InSSAPs were investigated. Characteristic colonoscopic findings were as follows: 1) color (normal, white, and red); 2) adherent mucous on the surface of the lesions either present or absent; 3) location either on the right (cecum to transverse colon) or the left side (descending colon to rectum) of the colon; 4) varicose microvascular vessel (VMV) [14] either present or absent using narrow band imaging with magnification ( $\bullet$ Fig. 1b); 5) expanded type II (E-II) pit pattern either present or absent ( $\boldsymbol{F}$ Fig. 2b) (the standard protocol for diagnosing an expanded type II pit pattern was the presence of a type II pit pattern with a modified Kudo type II pit pattern. These pits were wider and more rounded in shape, reflecting the dilatation of the crypts. We performed chromoendoscopy with indigo carmine dye spraying and crystal violet staining for all specimens) $[15,16]$;

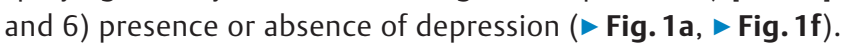
Endoscopically, the depression was deep and clearly demarcated.

\section{Identification of gene mutations}

We reviewed pathology reports from our hospital from January 2010 to June 2015 and abstracted representative cases of OSSA/Ps and InSSA/Ps and specimens that were in good condition. As a result, seven O-SSA/Ps and 11 InSSA/Ps specimens were usable, and O-SSA/Ps and InSSA/Ps were analyzed to iden-
- Table 1 SSA/P: 104 lesions (85 patients).

\begin{tabular}{|l|l|l|}
\hline & $\mathbf{N}=\mathbf{1 0 4}$ & $\%$ \\
\hline O-SSA/P & 67 & 64.4 \\
\hline InSSA/P & 37 & 35.6 \\
\hline - Expansive growth Type (EGT) & 15 & 14.4 \\
\hline - Infiltrating growth Type (IGT) & 22 & 21.2 \\
\hline
\end{tabular}

tify any significant differences in mutation rates. Genomic DNA was obtained from these tissues and sent to a research institution (MBL, Nagoya, Japan) for evaluation. Unstained sections, 10 - $\mu \mathrm{m}$ thick, were obtained from the formalin-fixed and paraffin-embedded tissue block. Thirty-six mutations in KRAS codon 61 (Q61K, Q61E, Q61 L, Q61P, Q61 R, and Q61H); KRAS codon 146 (A146T, A146S, A146P, A146E, A146V, and A146G); BRAF codon 600 (V600E); NRAS codon 12 (G12S, G12C, G12R, G12 D, G12V, and G12A); NRAS codon 13 (G13S, G13C, G13R, G13 D, G13V, and G13A); NRAS codon 61 (Q61K, Q61E, Q61L, Q61P, Q61R, and Q61H); PIK3CA exon 9 codon 542 (E542K); PIK3CA exon 9 codon 545 (E545K); PIK3CA exon 9 codon 546 (E546K); and PIK3CA exon 20 codon 1047 (H1047R and $\mathrm{H} 1047 \mathrm{~L}$ ) were analyzed using Luminex technology (GENOSEARCH Mu-PACK; MBL) [17].

\section{Statistical analyses}

Baseline characteristics of each case were compared using the $\mathrm{X}^{2}$ or Fisher's exact test for categorical data. The Mann-Whitney $U$ test was used for non-normally distributed continuous variables. $P<0.05$ was considered statistically significant. All analyses were performed using SPSS Version 22.0 (SPSS Inc., Chicago, Illinois, United States).

\section{Results}

We found the 104 lesions obtained from 85 patients. As shown in $>$ Table 1, 37 lesions were diagnosed as InSSA/Ps (35.6\%). All InSSA/Ps were divided into two growth patterns: EGT (14.4\%) and IGT $(21.2 \%)$.

- Table 2 shows the InSSA/P and O-SSA/P characteristics. In univariate analysis, male sex and presence of a depression were significantly associated with InSSA/Ps $(P<0.001)$. Median age of the patients tended to be older and tumor size tended to be smaller in InSSA/Ps, but these variables were not statistically significant. Presence of adherent mucous, frequency of occurrence in the right-side colon, a dilated type-II pit pattern, and presence of a varicose microvascular vessel were not different between InSSA/Ps and O-SSA/Ps.

- Table 3 shows characteristics of EGT and IGT types. A depression was seen in the EGT types (13/15) but not in the IGT type (0/22). Both EGT and IGT were seen more predominantly in men. Median age of patients with EGT and IGT was 70 and 71 years, respectively. EGT sizes $(9 \mathrm{~mm})$ were almost the same as those in IGT $(10 \mathrm{~mm})$. Presence of adherent mucous, frequency of occurrence in the right-side colon, E-II pit pattern, 
- Table 2 Characteristics of InSSA/P and O-SSA/P.

\begin{tabular}{|c|c|c|c|}
\hline & $\operatorname{InSSA} / \mathrm{P}$ & O-SSA/P & $P$ value \\
\hline \multicolumn{4}{|l|}{ Gender (\%) } \\
\hline " Male & $24(82.8)$ & $31(55.4)$ & 0.009 \\
\hline - Female & $5(17.2)$ & $25(44.6)$ & \\
\hline Median age (range), years & $70(53-79)$ & $67(29-83)$ & 0.071 \\
\hline \multicolumn{4}{|l|}{ Color (\%) } \\
\hline - Normal & $15(40.5)$ & $23(34.3)$ & 0.747 \\
\hline - White (Discolored) & $17(45.9)$ & $36(53.7)$ & \\
\hline - Red & $5(13.6)$ & $8(12.0)$ & \\
\hline \multicolumn{4}{|l|}{ Adherent mucous (\%) } \\
\hline - Positive & $33(89.1)$ & $58(86.6)$ & 0.768 \\
\hline - Negative & $4(10.9)$ & $9(13.4)$ & \\
\hline \multicolumn{4}{|l|}{ Location (\%) } \\
\hline - Right-side & $27(73.0)$ & $56(83.6)$ & 0.197 \\
\hline - Left-side & $10(17.0)$ & $11(16.4)$ & \\
\hline \multicolumn{4}{|l|}{ E-Il pit pattern ${ }^{1}(\%)$} \\
\hline - Positive & $20(54.1)$ & $37(55.2)$ & 0.415 \\
\hline - Negative & $8(21.6)$ & $20(29.9)$ & \\
\hline - Evaluation impossible & $9(24.3)$ & $10(14.9)$ & Spalte 4 \\
\hline \multicolumn{4}{|l|}{ VMV2 (\%) } \\
\hline " Positive & $28(75.7) 2$ & $39(58.2)$ & 0.053 \\
\hline - Negative & $3(8.1)$ & $19(28.4)$ & \\
\hline - Evaluation impossible & $6(16.2)$ & $9(13.4)$ & \\
\hline Tumor size, median (range), mm & $10(4-40)$ & $9(4-48)$ & 0.534 \\
\hline \multicolumn{4}{|l|}{ Depression (\%) } \\
\hline - Positive & $13(45.9)$ & $0(0.0)$ & $<0.001$ \\
\hline - Negative & $24(54.1)$ & $67(100)$ & \\
\hline
\end{tabular}

VMV, and presence of a depression were visibly similar between the EGT and IGT types.

The detected gene mutations are listed in $>$ Table 4 . A BRAF gene mutation at codon 600 of exon 15 (V600E) was found in six of seven O-SSA/Ps, in all five EGTs, and in all six IGTs; however, KRAS, NRAS, and PIK3CA mutations were not found in OSSA/Ps, EGT, or IGT.

\section{Discussion}

Compared with O-SSA/Ps, InSSA/Ps were more predominantly observed in men and in older patients. Therefore, it was thought that InSSA/Ps might have a different pathogenesis from O-SSA/Ps; therefore, we investigated the genetic features of InSSA/Ps and compared them with O-SSA/Ps. However, BRAF codon 600 mutations and KRAS, NRAS, and PIK3CA mutation status were similar between O-SSA/Ps and InSSA/Ps. Therefore, InSSA/Ps were thought to be a subtype of O-SSA/Ps.

The malignant potential of InSSA/Ps remains obscure. However, in our study, because InSSA/Ps have the same subtype as $\mathrm{O}-\mathrm{SSA} / \mathrm{Ps}$, prevalence of carcinomas in InSSA/Ps does not seem to be exceptional. The rapid progression of carcinoma arising from SSA/Ps has already been suggested $[14,18,19]$. Histologically, InSSA/Ps with invagination into the submucosal layer might induce rapid progression to CRCs. There were two case reports regarding carcinoma arising in InSSA/P [20,21]. Therefore, we might be able to prevent rapid progression to CRCs by removing InSSA/Ps. InSSA/Ps accounted for $35.6 \%$ of all SSA/Ps 
- Table 3 Characteristics of EGT and IGT.

\begin{tabular}{|c|c|c|c|c|}
\hline & InSSA/P & EGT & IGT & $P$ value \\
\hline \multicolumn{5}{|l|}{ Gender (\%) } \\
\hline - Male & $24(82.8)$ & $10(83.3)$ & $14(82.4)$ & $\mathrm{NS}^{1}$ \\
\hline - Female & $5(17.2)$ & $2(16.7)$ & $3(17.6)$ & \\
\hline Median age (range), years & $70(53-79)$ & $71(61-84)$ & $70(53-77)$ & 0.237 \\
\hline \multicolumn{5}{|l|}{ Color (\%) } \\
\hline - Normal & $15(40.5)$ & $7(46.7)$ & $9(40.9)$ & 0.926 \\
\hline - White (Discolored) & $17(45.9)$ & $7(46.7)$ & $11(50.0)$ & \\
\hline - Red & $5(13.6)$ & $1(6.6)$ & $2(9.1)$ & \\
\hline \multicolumn{5}{|l|}{ Adherent mucous (\%) } \\
\hline - Positive & $33(89.1)$ & $14(93.3)$ & $19(86.4)$ & 0.633 \\
\hline - Negative & $4(10.9)$ & $1(6.7)$ & $3(13.6)$ & \\
\hline \multicolumn{5}{|l|}{ Location (\%) } \\
\hline - Right-side & $56(83.6)$ & $12(80.0)$ & $16(72.7)$ & 0.711 \\
\hline - Left-side & $11(16.4)$ & $3(20.0)$ & $6(27.3)$ & \\
\hline \multicolumn{5}{|l|}{ E-II pit pattern ${ }^{2}(\%)$} \\
\hline - Positive & $20(54.1)$ & $10(66.6)$ & $10(45.5)$ & $\mathrm{NS}^{2}$ \\
\hline - Negative & $8(21.6)$ & $3(20.0)$ & $4\left(\begin{array}{lll}18 & 1\end{array}\right)$ & \\
\hline - Evaluation impossible & $9(24.3)$ & $2(14.4)$ & $8(36.4)$ & \\
\hline \multicolumn{5}{|l|}{ VMV2 (\%) } \\
\hline - Positive & $28(75.7)$ & $13(87.4)$ & $15(68.2)$ & $\mathrm{NS}^{2}$ \\
\hline - Negative & $3(8.1)$ & $1(6.3)$ & $3(13.6)$ & \\
\hline - Evaluation impossible & $6(16.2)$ & $1(6.3)$ & $4(18.2)$ & \\
\hline Tumor size, median (range), mm & $10(4-40)$ & $9(4-40)$ & $10(4-35)$ & 0.963 \\
\hline \multicolumn{5}{|l|}{ Depression (\%) } \\
\hline - Positive & $17(45.9)$ & $13(86.7)$ & $0(0.0)$ & $<0.001$ \\
\hline - Negative & $20(54.1)$ & $2(13.3)$ & $22(100)$ & \\
\hline
\end{tabular}

- Table 4 Identification of gene mutation.

\begin{tabular}{|l|l|l|l|}
\hline & O-SSA/P (n=7) & EGT (n=5) & IGT (n=6) \\
\hline BRAF codon 600 & & \\
\hline - Mutation (+) & $6(82.8)$ & $5(100)$ & $6(100)$ \\
\hline - Mutation (-) & $1(17.2)$ & $0(0)$ & $0(0)$ \\
\hline
\end{tabular}

in this study. We speculate that inverted growth is a special finding, but is occasionally seen in SSA/Ps. Histologically, InSSA/Ps are defined as lesions with the cellular and tubular characteristics of SSA/Ps that grow in an endophytic manner. A sim- ilar phenomenon has been observed in HP and has been referred to as InHPs [12,22]. Based on growth pattern classification of InHPs, we categorized InSSA/Ps according to patterns of invasion into EGT and IGT types. All InSSA/Ps were successfully divided into the two growth patterns of EGT and IGT. InHP have been reported to occur predominantly on the right side of the colon and to be relatively large [20]. In this study, we also showed that most InSSAPs were also on the right side of the colon and had a median size of $10 \mathrm{~mm}$. Therefore, we speculate that some reported InHPs can be included as InSSA/Ps. A previous report described that as many as one-third of SSA/Ps could be erroneously classified as innocuous HPs by community gastroenterologists using the NICE classification [23]. 
In our study, endoscopic characteristics under magnifying colonoscopy of most InSSA/Ps were a dilated type-II pit pattern and irregularly dilated vessels. It has been reported that SSA/Ps were macroscopically either flat (Paris classification 0 - Ila and 0 - IIb) or sessile (Paris classification 0-Is) [14], whereas the EGT of InSSA/Ps included a depressed area (Paris classification $0-$ IIc). From the feature of the depression, an EGT type of InSSA/Ps can be diagnosed endoscopically. Owing to the inverted growth pattern, a depressed area is present in InSSA/P, which could be misdiagnosed as early colorectal neoplasm on using a conventional view. Therefore, it is important to diagnose the EGT of InSSA/Ps. IGT types of InSSA/Ps were the same macroscopic type for O-SSA/Ps. Therefore, it was difficult to diagnose endoscopically IGT types of InSSA/Ps. An endoscopic biopsy is not sufficient to make an accurate diagnosis of InSSA/P because muscularis mucosa and/or submucosal layer is not included with small specimens. Therefore, the final diagnosis of InSSA/ Ps depends on the pathological findings of endoscopic en bloc resection [24]. Furthermore, some InSSA/Ps resemble an inverted colonic diverticulum (ICD). Thus, there is a risk of misdiagnosis of InSSA/Ps, particular of the EGT type and, in the case of $I C D$, endoscopic removal can cause serious complications, such as perforation [25]. A dilated type-II pit pattern and an irregularly dilated vessel could be an endoscopic hallmark of InSSA/ Ps, which is different from ICD, which has a normal type-I pit pattern.

This study has some limitations. We identified SSA/P from the pathology reports. Therefore, the results are not representative of all SSA/Ps. However, because we removed all SSA/Ps endoscopically diagnosed in our institute, our results may be reasonable in the context of clinical evaluation of SSA/Ps. The small number of genomic analyses in our investigation appears to be another limitation. Due to the BRAF mutation, endoscopic images, and clinicopathological features, InSSA/Ps are thought to be a subtype of O-SSA/Ps owing to the limited number of genetic analyses performed in our hospital.

\section{Conclusion}

Although there are some limitations, more than $30 \%$ of SSAPs were pathologically diagnosed as InSSAPs in our practical endoscopic-resected specimens. Approximately $40 \%$ of InSSAPs were of the EGT type, which has a demarcated depression and is easily diagnosed with endoscopy. Both InSSA/Ps and O-SSA/ Ps had a high rate of BRAF mutation. However, there were no genetic findings to assess the malignant potential in InSSAPs in this study due to the limited number of studies performed in our hospital.

\section{Acknowledgements}

The study protocol was approved by the hospital's Medical Ethics Committee, by the National Cancer Center, and by the medical ethics committee of our hospital (2015-151) and was performed in accordance with the ethical principles outlined in the Declaration of Helsinki. Patient informed consent was waived due to the retrospective design of the study.

\section{Competing interests}

None

References

[1] Vogelstein B, Fearon ER, Hamilton SR et al. Genetic alterations during colorectal-tumor development. N Engl J Med 1988; 319: 525-532

[2] Snover DC. Update on the serrated pathway to colorectal carcinoma. Hum Pathol 2011; 42: 1- 10

[3] Erichsen R, Baron JA, Hamilton-Dutoit S] et al. Increased risk of colorectal cancer development among patients with serrated polyps. Gastroenterology 2016; 150: 895-902

[4] Torlakovic E, Skovlund E, Snover DC et al. Morphologic reappraisal of serrated colorectal polyps. Am J Surg Pathol 2003; 27: 65-81

[5] Goldstein NS, Bhanot P, Odish E et al. Hyperplastic-like colon polyps that preceded microsatellite-unstable adenocarcinomas. Am J Clin Pathol 2003; 119: $778-796$

[6] Higuchi T, Jass JR. My approach to serrated polyps of the colorectum. J Clin Pathol 2004; 57: 682 -686

[7] Kambara T, Simms LA, Whitehall VL et al. BRAF mutation is associated with DNA methylation in serrated polyps and cancers of the colorectum. Gut 2004; 53: $1137-1144$

[8] Okamoto K, Kitamura S, Kimura T et al. Clinicopathological characteristics of serrated polyps as precursors to colorectal cancer: Current status and management. J Gastroenterol Hepatol 2017; 32: 358-367

[9] O'Brien M], Yang S, Mack C et al. Comparison of microsatellite instability, CpG island methylation phenotype, BRAF and KRAS status in serrated polyps and traditional adenomas indicates separate pathways to distinct colorectal carcinoma end points. Am J Surg Pathol 2006; 30: 1491 - 1501

[10] Snover DC, Ahnen DJ, Burt RW et al. Serrated polyps of the colon and rectum and serrated polyposis. WHO classification of tumours of the digestive system In: Bosman FT, Carneiro F, Hruban RH et al. editors 4th ed. Lyon: IARC; 2010

[11] Muramoto T, Oono Y, Fu KI et al. Inverted sessile serrated polyp diagnosed by magnifying image-enhanced colonoscopy. Endoscopy 2011; 43: $201-202$

[12] Sobin LH. Inverted hyperplastic polyps of the colon. Am J Surg Pathol 1985; 9: 265-272

[13] Yantiss RK, Goldman H, Odze RD. Hyperplastic polyp with epithelial misplacement (inverted hyperplastic polyp): a clinicopathologic and immunohistochemical study of 19 cases. Mod Pathol 2001; 14: 869875

[14] Uraoka T, Higashi R, Horii J et al. Prospective evaluation of endoscopic criteria characteristic of sessile serrated adenomas/polyps. J Gastroenterol 2015; 50: 555-563

[15] Kimura T, Yamamoto E, Yamano HO et al. A novel pit pattern identifies the precursor of colorectal cancer derived from sessile serrated adenoma. Am J Gastroenterol 2012; 107: 460-469

[16] Kudo S, Tamura S, Nakajima T et al. Diagnosis of colorectal tumorous lesions by magnifying endoscopy. Gastrointest Endosc 1996; 44: $1653-1664$

[17] Sano Y, Ikematsu H, Fu KI et al. Meshed capillary vessels by use of narrow-band imaging for differential diagnosis of small colorectal polyps. Gastrointest Endosc 2009; 69: 278-283

[18] Oono Y, Fu K, Nakamura H et al. Progression of a sessile serrated adenoma to an early invasive cancer within 8 months. Dig Dis Sci 2009; 54: 906 - 909 
[19] Mäkinen M]. Colorectal serrated adenocarcinoma. Histopathology 2007; 50: $131-150$

[20] Fu K, Fujii T, Kuwayama $\mathrm{H}$ et al. Invasive cancer arising in a colonic inverted hyperplastic polyp. Endoscopy 2009; 42: 29-30

[21] Hyun Ho C, Sung-Ha B, Eun-Chul J et al. Intramucosal colon cancer developing in an inverted hyperplastic polyp. Gut Liver 2015; 7: 126 127

[22] Shepherd NA. Inverted hyperplastic polyposis of the colon. J Clin Pathol 1993; 46: $56-60$
[23] Singh A, Konda V], Siddiqui UD. The not so NICE classification. Gastrointest Endosc 2014; 79: 1030 - 1031

[24] Hirasaki S, Kanzaki H, Suzuki S et al. Pedunculated inverted hyperplastic polyp of the sigmoid colon treated with endoscopic polypectomy. Dig Endosc 2009; 21: 275-276

[25] Yusuf SI, Grant C. Inverted colonic diverticulum: a rare finding in a common condition? Gastrointest Endosc 2000; 52: 111-115 